\title{
BMJ Open Differences in rates and odds for emergency caesarean section in six Palestinian hospitals: a population- based birth cohort study
}

\author{
Mohammed Zimmo, ${ }^{1,2,3}$ Katariina Laine, ${ }^{4,5}$ Sahar Hassan, ${ }^{6}$ Erik Fosse, ${ }^{2,3}$ \\ Marit Lieng, ${ }^{2,7}$ Hadil Ali-Masri, ${ }^{2,3,8}$ Kaled Zimmo, ${ }^{2,3,9}$ Marit Anti, ${ }^{10}$ Bettina Bottcher, ${ }^{11}$ \\ Ragnhild Sørum Falk, ${ }^{12}$ Åse Vikanes ${ }^{3}$
}

To cite: Zimmo M, Laine $\mathrm{K}$, Hassan S, et al. Differences in rates and odds for emergency caesarean section in six Palestinian hospitals: a population-based birth cohort study. BMJ Open 2018;8:e019509. doi:10.1136/ bmjopen-2017-019509

- Prepublication history and additional material for this paper are available online. To view these files, please visit the journal online (http://dx.doi. org/10.1136/bmjopen-2017019509).

Received 6 September 2017 Revised 4 February 2018 Accepted 9 February 2018

Check for updates

For numbered affiliations see end of article.

\section{Correspondence to} Dr Mohammed Zimmo; mohammedzimm01983@gmail. com

\section{ABSTRACT}

Objective To assess the differences in rates and odds for emergency caesarean section among singleton pregnancies in six governmental Palestinian hospitals. Design A prospective population-based birth cohort study. Setting Obstetric departments in six governmental Palestinian hospitals.

Participants 32321 women scheduled to deliver vaginally from 1 March 2015 until 29 February 2016. Methods To assess differences in sociodemographic and antenatal obstetric characteristics by hospital, $\chi^{2}$ test, analysis of variance and Kruskal-Wallis test were applied. Logistic regression was used to estimate differences in odds for emergency caesarean section, and ORs with $95 \%$ Cls were assessed.

Main outcome measures The primary outcome was the adjusted ORs of emergency caesarean section among singleton pregnancies for five Palestinian hospitals as compared with the reference (Hospital 1).

Results The prevalence of emergency caesarean section varied across hospitals, ranging from $5.8 \%$ to $22.6 \%$ among primiparous women and between $4.8 \%$ and $13.1 \%$ among parous women. Compared with the reference hospital, the ORs for emergency caesarean section were increased in all other hospitals, crude ORs ranging from 1.95 (95\% Cl 1.42 to 2.67$)$ to 4.75 (95\% Cl 3.49 to 6.46$)$ among primiparous women. For parous women, these differences were less pronounced, crude ORs ranging from 1.37 (95\% Cl 1.13 to 1.67$)$ to 2.99 (95\% Cl 2.44 to 3.65$)$. After adjustment for potential confounders, the ORs were reduced but still statistically significant, except for one hospital among parous women.

Conclusion Substantial differences in odds for emergency caesarean section between the six Palestinian governmental hospitals were observed. These could not be explained by the studied sociodemographic or antenatal obstetric characteristics.

\section{INTRODUCTION}

Caesarean section is one of the most common surgical procedures worldwide. ${ }^{1}$ The rate of caesarean section has increased globally from $7 \%$ in 1990 to $19 \%$ in $2014 .^{2}$ This increase in
Strengths and limitations of this study

- This study is the largest, population-based, prospective birth cohort study in Palestine, which includes both Gaza and West Bank hospitals.

- All singleton pregnant women aiming to give birth vaginally in the six study hospitals were included, reducing the risk for selection bias.

- The main limitation of this study was the large proportion of missing data on mode of deliveries in one of the study hospitals. The missing data were expected to be random and therefore not influencing the exposure-outcome associations studied.

- Data on diabetes before and during pregnancy were not registered accurately and could therefore not be used for analyses.

- There was an inaccurate registration of maternal weight and place of residence in some hospitals.

caesarean section rates is, however, not associated with improved outcomes for mothers and newborns. ${ }^{3}$ Although delivery by caesarean section is considered safe, it is associated with adverse short-term as well as long-term consequences for mothers and children. ${ }^{4}$ The most worrying rise in caesarean section rate is therefore seen among healthy primiparous women with singleton pregnancies at term, who were having a low risk of caesarean section. This rise was significantly higher in women who underwent induction of labour. ${ }^{5}$

Despite international evidence-based guidelines for indications of caesarean section, the caesarean section rates vary between countries (from 5\% in Sub-Saharan Africa to around $40 \%$ in Latin America) and even between hospitals within the same countries. $^{26}$ A study found that different caesarean section rates in National Health Service (NHS) trusts in the UK were mainly restricted to emergency caesarean section 
and not to planned caesarean sections. ${ }^{6}$ Another study reported that differences in maternal or fetal risk factors do not explain the variations in caesarean section rates. ${ }^{7}$ Healthcare professionals' decision to perform caesarean section is known to be influenced by cultural factors, legal liability as well as medical evidence. ${ }^{8}$

In Palestine, two previous studies on caesarean section rates have been published. ${ }^{9}{ }^{10}$ A study from Makassed Charitable Hospital in East Jerusalem, including 6804 women, showed that the caesarean section rate increased from $9.4 \%$ to $14.4 \%$ between 1993 and 2002. ${ }^{10}$ Another study in 2006, using data from the Palestinian Family Health Survey of 6113 women from Gaza and the West Bank, reported an increase in caesarean section rates from $6.0 \%$ in 1996 to $14.8 \%$ in $2006 .{ }^{9}$ Both studies lacked information on potential confounders. According to the Palestinian Ministry of Health in 2015, the overall caesarean section rate was $23.2 \% .^{11}$ Since these studies were published, the political situation in the occupied Palestinian Territories has become more challenging given the siege on Gaza, which was recently reported to influence health services. ${ }^{12}$

The main aim of this study was to explore any differences in rates and odds for emergency caesarean section among singleton pregnancies in six governmental hospitals in Palestine.

\section{METHODS}

The data were obtained from a population-based birth cohort study in six Palestinian governmental hospitals from 1 March 2015 until 29 February 2016. Three hospitals (1, 2 and 3$)$ were located in Gaza and three (4, 5 and 6 ) in the West Bank. All hospitals were teaching hospitals except one (Hospital 2). Teaching hospitals in Palestine have educational programmes for health personnel, such as nurses, midwives and medical doctors. All were referral hospitals, except one (Hospital 1). Referral hospitals in Palestine receive patients from other governmental or private hospitals in the neighbouring areas. Hospital 1, being non-referral, was the only one without a maternal intensive care unit.

All women planned for vaginal delivery were included in the study. Women planned for elective caesarean section, those with two or more previous caesarean sections, multiple gestations and those with missing information about the actual mode of delivery were excluded from the study sample (see online supplementary figure 1).

\section{Data collection and entry}

A case registration form, developed by Palestinian and Norwegian obstetricians and midwives, was used to collect data on maternal sociodemographic, antenatal obstetric characteristics and mode of delivery prospectively. ${ }^{13}$ Before the data collection started, research teams in each hospital were established, comprising the heads of obstetric departments, medical doctors and midwives working in the labour wards. The case registration form was filled in by doctors and midwives attending the births. The registered data were entered by research teams into a tailor-made version of District Health Information Software 2 (DHIS 2, V.2.24). DHIS 2 has been created by the Department of Global Infrastructure at the University of Oslo. It is a free, adaptable web-based open-source information system tool developed with support from the Norwegian Agency for Development. Data were transferred from DHIS 2 to be stored in Service for Sensitive Data (TSD) platform, which is developed and operated by the University of Oslo for researchers to collect, store, analyse and share sensitive data in compliance with the Norwegian regulations regarding individuals' privacy ( tsd-drift@usit.uio.no).

\section{Risk factors}

Data on maternal pre-pregnancy weight and height were obtained from the mother and child health handbook, and if the booklet was unavailable, the medical teams obtained this information by asking the women.

Maternal age was categorised into 5-year age groups (table 1). Place of residence was dichotomised into camp or urban-rural area. Maternal education was categorised into three groups according to length of education (table 1). Pre-pregnancy body mass index was categorised according to the WHO classification: $\leq 18.5,18.5-$ 24.9, $25.0-29.9$ and $\geq 30.0 \mathrm{~kg} / \mathrm{m}^{2}$. ${ }^{14}$ Mode of delivery was dichotomised into vaginal (normal and assisted vaginal) and emergency caesarean section.

Parity was dichotomised into primiparous and parous women. Primiparous women have had no previous delivery, whereas parous women had one or more previous deliveries. Previous caesarean section was dichotomised into no/yes. Number of antenatal visits was categorised into four groups: $<3,4-7$ and $\geq 8$ visits. In vitro fertilisation treatment was dichotomised into no/ yes. Hypertensive disorder, which included hypertension before as well as during pregnancy and pre-eclampsia, was dichotomised into no/yes. Induction of labour, by misoprostol or balloon catheter, was dichotomised into no/yes. Unknown information of hypertensive disorder and induction of labour were considered as no disorder/ induction.

\section{Outcomes}

The primary outcome was the adjusted ORs of emergency caesarean section among singleton pregnancies for five Palestinian hospitals as compared with the reference (Hospital 1).

Emergency caesarean section covered a wide range of clinical situations from an immediate threat to the mother or baby to conditions requiring early delivery. The criteria for emergency caesarean section in this study reflect Lucas urgency classification one, two and three. ${ }^{15}$

\section{Statistical analysis}

Descriptive analyses were conducted for the baseline characteristics of the women. To assess differences by 
Table 1 Sociodemographic characteristics of the study population ( $N=32321)$

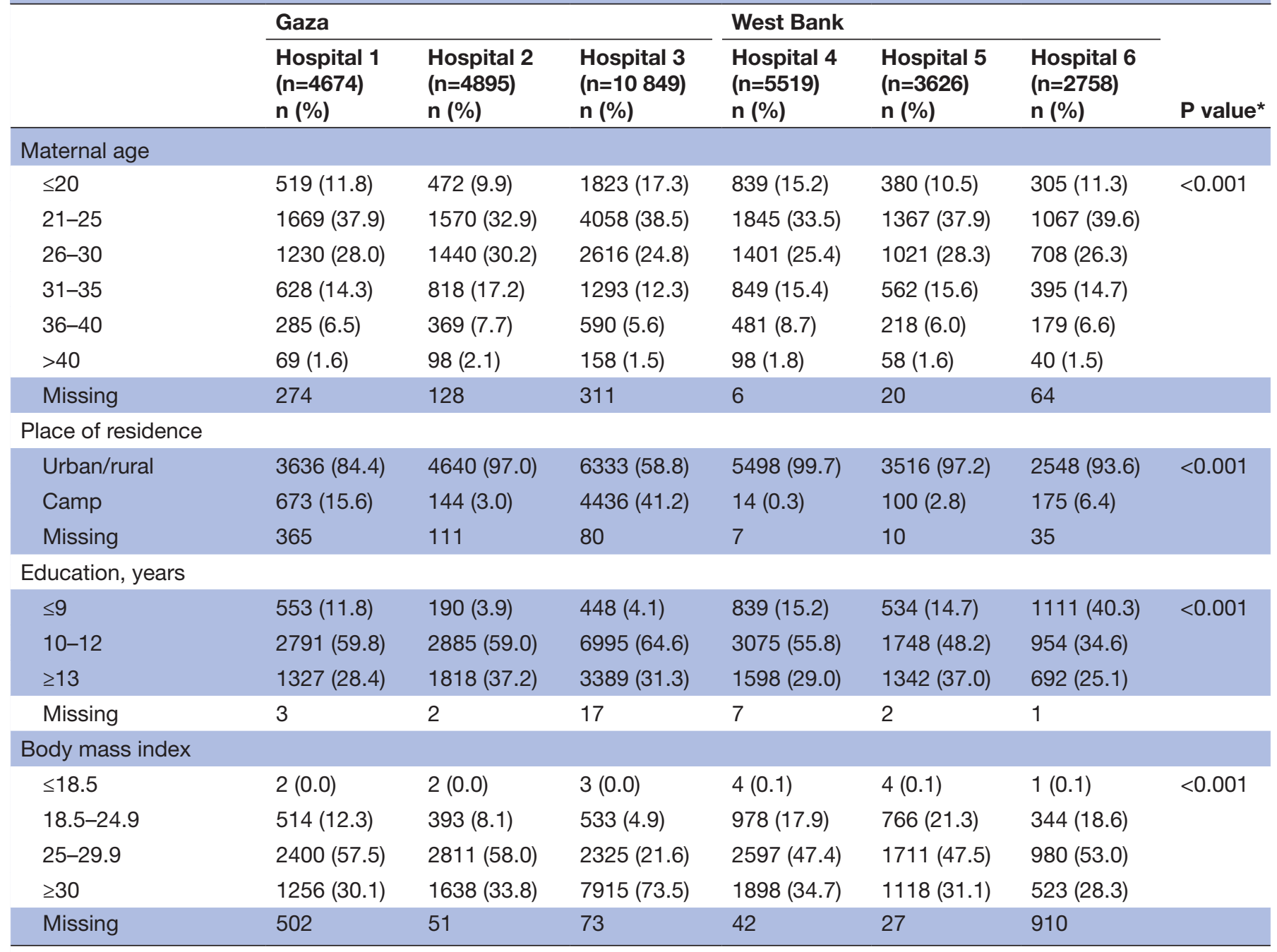

${ }^{*} \mathrm{P}$ value from $\chi^{2}$ test is used to test the difference between hospitals.

hospitals, comparison of proportions was tested by $\chi^{2}$ test, differences in means by one-way analysis of variance and differences in median by Kruskal-Wallis test.

In order to study the effect of the hospital on the odds of emergency caesarean section, logistic regression analyses were applied, stratified according to parity. Sociodemographic characteristics (age, place of residence, education, body mass index) and antenatal obstetric characteristics (number of children alive, previous caesarean section, number of antenatal visits, in vitro fertilisation treatment, hypertensive disorder and induction of labour) were included as potential confounders. Three separate models were performed. Model 1 assessed the influence of sociodemographic characteristics, Model 2 antenatal obstetric characteristics and Model 3 included both sociodemographic and antenatal obstetric characteristics combined. The strength of the association between each variable and the odds of emergency caesarean section was estimated by ORs with $95 \%$ CIs. Due to low numbers in categories, $\leq 18.5$ and $18.5-24.9 \mathrm{~kg} / \mathrm{m}^{2}$ of body mass index, they merged into one group in regression analyses.
The proportion of missing data was low $(<5 \%)$; therefore, multiple imputation was not considered.

Multicollinearity of independent variables was checked via the variance inflation factor statistic. For primiparous women, education had to be excluded due to multicollinearity. We found no multicollinearity among parous women. Interaction between hospital and the adjusting variables were explored by entering product terms, one at a time, into the model. Interactions with $\mathrm{P}<0.001$ were reported in the text.

$P$ values $<0.05$ were considered statistically significant. All statistical analyses were performed using SPSS V.22 (SPSS, Chicago, Illinois, USA).

\section{RESULTS}

During the study period, 35109 women gave birth. In total, 32321 singleton pregnant women were planned for vaginal birth and included in this study. Of these women, $2932(9.1 \%)$ were delivered by emergency caesarean section. 
Table 2 Antenatal obstetric characteristics of the study population ( $N=32321)$

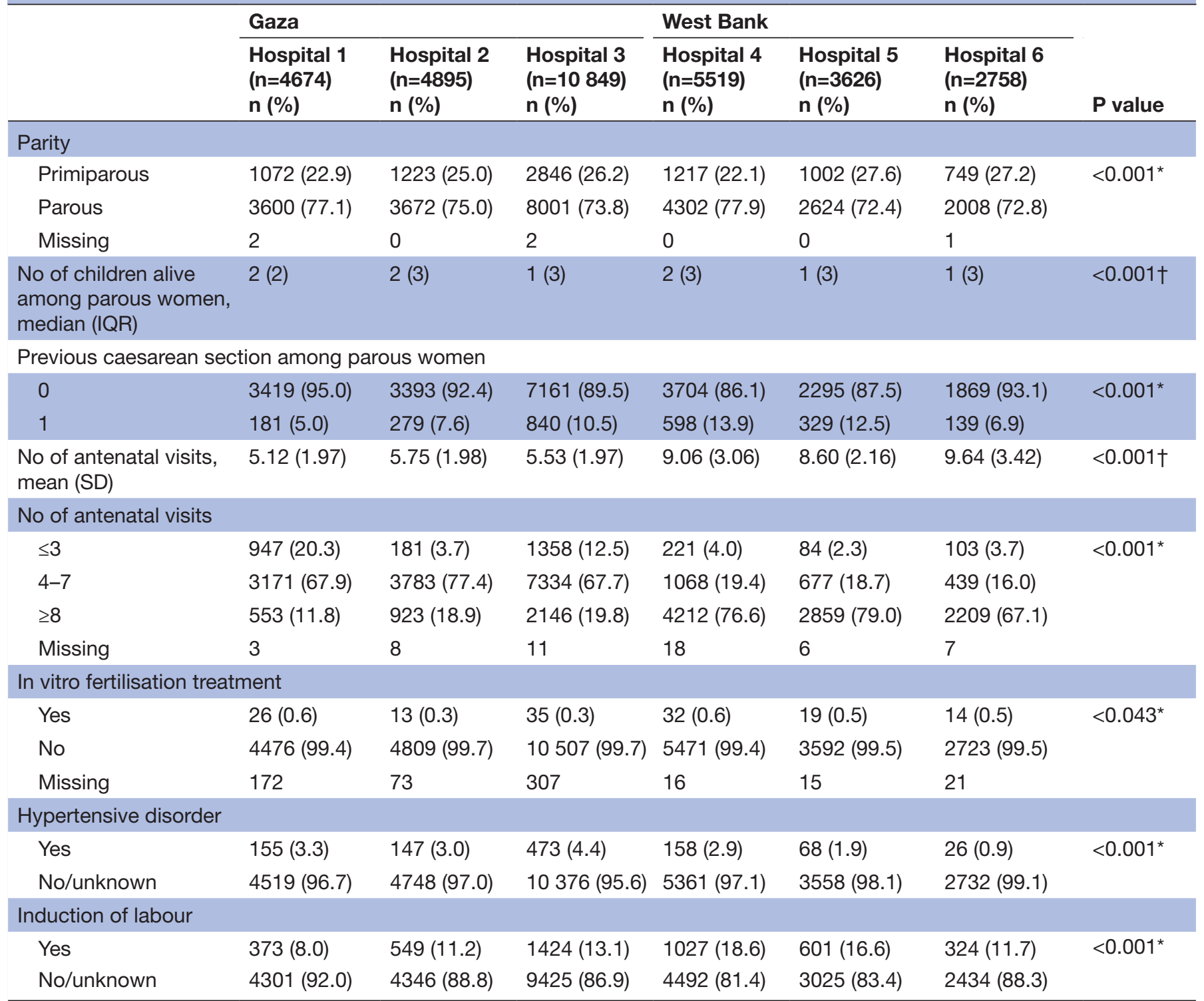

${ }^{*} \mathrm{P}$ value from $\chi^{2}$ test is used to test the difference between hospitals.

†P value from analysis of variance is used to test the difference between hospitals.

Significant differences were found between the hospitals for maternal age, place of residence, education and body mass index, all $\mathrm{P}$ values $<0.001$. Hospital 3 had the largest proportion of the youngest women giving birth with $17.3 \%$ being 20 years old or younger. In contrast, Hospital 2 had the largest proportion of the oldest women delivering, where approximately $2 \%$ were aged 40 years or more.

There were significant differences between hospitals regarding women living in refugee camps ranging from $0.3 \%$ in Hospital 4 to $41.2 \%$ in Hospital 3. Almost $60 \%$ had between 10 and 12 years of education, ranging from $34.6 \%$ in Hospital 6 to $64.6 \%$ in Hospital 3. More than $80 \%$ (27 172) of the women had a body mass index $\geq 25 \mathrm{~kg} / \mathrm{m}^{2}$ (table 1).

Table 2 describes the antenatal obstetric characteristics of all births. About $25 \%$ of women were primiparous.
Furthermore, women in Hospitals 4 and 5 had significantly more induction of labour than other hospitals (table 2). There were significant differences in hypertensive disorders between hospitals ranging from $0.9 \%$ in Hospital 6 to $4.4 \%$ in Hospital 3. The numbers of antenatal visits varied between hospitals. The women in Hospitals 1, 2 and 3 in Gaza had around five antenatal visits (SD 2.0; range 0-24) compared with nine (SD 2.9; range 0-29) in Hospitals 4, 5 and 6 in the West Bank (table 2). Less than $1 \%$ of women in all hospitals had undergone in vitro fertilisation treatment.

Significant differences in the prevalence of emergency caesarean section between hospitals were observed, for primiparous as well as parous women. Among primiparous women, the prevalence was $12.4 \%$, ranging from $5.8 \%$ in Hospital 1 to $22.6 \%$ in Hospital 6. Likewise, for parous women, the prevalence was $7.9 \%$, ranging from $4.8 \%$ in Hospital 1 to $13.1 \%$ in Hospital 6 (table 3). 
Table 3 Prevalence of emergency caesarean section in the study hospitals

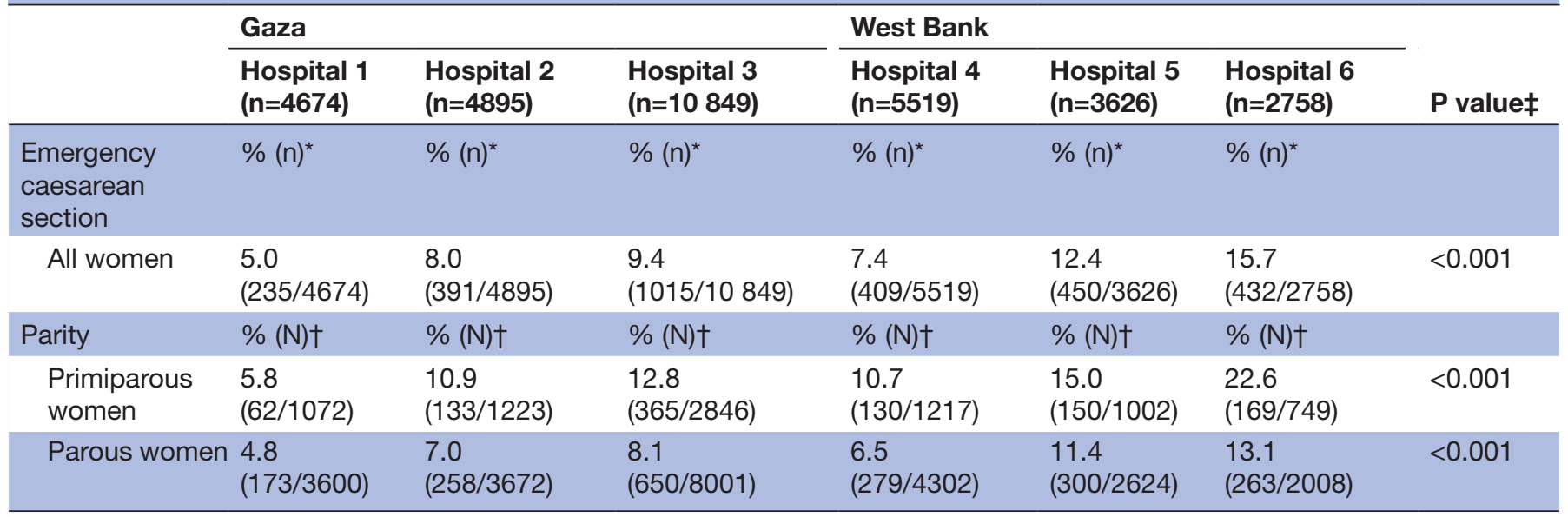

${ }^{*} \mathrm{~N}=$ number of emergency caesarean section/total number of deliveries in the hospital.

$\dagger \mathrm{N}=$ number of emergency caesarean section/total number of deliveries among women group in the hospital. $\ddagger \mathrm{P}$ value from $\chi^{2}$ test.

Among primiparous women, the ORs for emergency caesarean section differed by hospital (table 4). Hospital 1 had the lowest prevalence of emergency caesarean section and was thus considered the reference hospital. The largest difference was found for Hospital 6, crude OR 4.75 (95\% CI 3.49 to 6.46$)$. When adjusting for potential confounders, the ORs for different hospitals were reduced, but still statistically significant (table 4, model 3). When checking for interaction, the body mass index modified the effect of hospitals. In Hospital 3, the OR decreased with increasing body mass index (body mass index $\geq 30$ : OR 0.31 , 95\% CI 0.11 to 0.90), whereas the other hospitals had increased ORs with increasing body mass index.

A similar trend was found among parous women, but the differences between hospitals were less pronounced compared with primiparous women (table 5). After including sociodemographic and antenatal obstetric confounders in the model, the difference between the hospitals was less clear but still statistically significant for all hospitals, except Hospital 4. The strongest risk factor for emergency caesarean section was previous caesarean section (OR 6.26, 95\% CI 5.57 to 7.05 ).

Interactions by hospital were observed for previous caesarean section and for hypertensive disorder among parous women. The OR of emergency caesarean section was 15-17 for women with previous caesarean section compared with no previous caesarean section in Hospitals 2 and 6 , whereas the other hospitals had a corresponding OR of 4-8. Furthermore, an OR of 10 was observed for women with hypertensive disorder compared with those without hypertensive disorder in Hospital 1, whereas the other hospitals had a corresponding OR of 2-4 of emergency caesarean section.

\section{DISCUSSION}

There were notable variations between study hospitals in rate and odds for emergency caesarean section among singleton pregnancies for primiparous and parous women. Compared with the hospital with the lowest prevalence for emergency caesarean section, the crude ORs were increased in all other hospitals by up to almost fivefold for the primiparous and threefold for parous women. The effect of sociodemographic and maternal antenatal obstetrical characteristics in odds for emergency caesarean section is in line with previous research, confirming previous caesarean section, hypertension disorder and in vitro fertilisation treatment as the strongest risk factors for emergency caesarean section. ${ }^{16} 17$ So far, this is the largest birth cohort study in Palestine, including 32321 women, and the first to focus on emergency caesarean section.

Caesarean section may be life-saving for both the mother and the newborn, but overuse of caesarean section does not improve maternal or perinatal health. ${ }^{418}$ Immediate surgical complications and adverse effects in future pregnancies, such as increased risk of intrauterine fetal demise, preterm delivery, uterine abruption and abnormal placentation (praevia, accreta, increta), are reasons why caesarean section should be performed when clinically indicated only. ${ }^{419}$ Increasing numbers of repeat caesarean sections increases the risk of severe complications on the individual level. Knowing that a previous caesarean section increases the risk for repeat caesarean section in the next pregnancy ${ }^{20}$ makes the management of the delivery of a primiparous woman a true challenge in obstetrics.

Large differences in caesarean section rates between hospitals may reflect varying skills and working methods. ${ }^{21}$ There are few previous studies on differences in caesarean section rates and risk between hospitals within the same country. A large study from England compared 146 NHS trusts, including 620604 singleton births. ${ }^{6}$ The study showed that unadjusted rates of caesarean sections among the NHS trusts differed notably, ranging from 
Table 4 OR and 95\% Cls for emergency caesarean section among primiparous women in the participating hospitals $(\mathrm{N}=8109)$

$\begin{array}{llll}\text { Crude OR } & \text { Model } 1^{*} & \text { Model } 2 \dagger & \text { Model } 3 \ddagger \\ (95 \% \mathrm{Cl}) & \text { OR }(95 \% \mathrm{Cl}) & \text { OR }(95 \% \mathrm{Cl}) & \text { OR }(95 \% \mathrm{Cl})\end{array}$

\begin{tabular}{|c|c|c|c|c|}
\hline \multicolumn{5}{|l|}{ Hospitals } \\
\hline Hospital 1 & Ref & Ref & Ref & Ref \\
\hline Hospital 2 & 1.99 (1.45 to 2.72$)$ & 1.90 (1.34 to 2.70$)$ & 1.99 (1.44 to 2.75$)$ & 1.87 (1.30 to 2.68$)$ \\
\hline Hospital 3 & 2.40 (1.81 to 3.17$)$ & 2.40 (1.73 to 3.33$)$ & 2.43 (1.82 to 3.24$)$ & 2.47 (1.77 to 3.46$)$ \\
\hline Hospital 4 & 1.95 (1.42 to 2.67$)$ & 2.33 (1.64 to 3.31$)$ & 1.58 (1.11 to 2.25$)$ & 1.84 (1.24 to 2.73$)$ \\
\hline Hospital 5 & 2.87 (2.11 to 3.91$)$ & 2.99 (2.12 to 4.22$)$ & 2.49 (1.77 to 3.50$)$ & 2.53 (1.74 to 3.70$)$ \\
\hline Hospital 6 & 4.75 (3.49 to 6.46$)$ & 4.28 (2.94 to 6.22$)$ & 4.11 (2.87 to 5.90$)$ & 3.54 (2.29 to 5.47$)$ \\
\hline Maternal age (years) & 1.12 (1.10 to 1.13$)$ & $1.12(1.10$ to 1.14$)$ & & $1.12(1.10$ to 1.14$)$ \\
\hline \multicolumn{5}{|l|}{ Place of residence } \\
\hline Urban/rural & Ref & Ref & & Ref \\
\hline Camp & $1.22(1.04$ to 1.44$)$ & 1.32 (1.08 to 1.61$)$ & & 1.24 (1.01 to 1.53$)$ \\
\hline \multicolumn{5}{|l|}{ Education (years) } \\
\hline$\leq 9$ & Ref & NA & & NA \\
\hline 10 to 12 & 0.50 (0.41 to 0.62$)$ & & & \\
\hline$\geq 13$ & 0.58 (0.47 to 0.72$)$ & & & \\
\hline \multicolumn{5}{|c|}{ Body mass index $\left(\mathrm{kg} / \mathrm{m}^{2}\right)$} \\
\hline$\leq 24.9$ & Ref & Ref & & Ref \\
\hline 25 to 29.9 & 1.15 (0.91 to 1.46$)$ & 1.17 (0.92 to 1.50$)$ & & 1.12 (0.88 to 1.44$)$ \\
\hline$\geq 30$ & 1.50 (1.20 to 1.89$)$ & 1.41 (1.10 to 1.81$)$ & & 1.30 (1.01 to 1.68$)$ \\
\hline Antenatal visits (no) & 1.07 (1.05 to 1.10$)$ & & 1.04 (1.01 to 1.07$)$ & 1.04 (1.01 to 1.07$)$ \\
\hline \multicolumn{5}{|c|}{ In vitro fertilisation treatment } \\
\hline No & Ref & & Ref & Ref \\
\hline Yes & 5.93 (3.61 to 9.75$)$ & & 6.62 (3.97 to 1.05 ) & 4.64 (2.66 to 8.08$)$ \\
\hline \multicolumn{5}{|l|}{ Hypertensive disorder } \\
\hline No/unknown & Ref & & Ref & Ref \\
\hline Yes & 3.62 (2.75 to 4.77$)$ & & 3.95 (2.96 to 5.28 ) & 3.56 (2.61 to 4.86$)$ \\
\hline \multicolumn{5}{|l|}{ Induction of labour } \\
\hline No/unknown & Ref & & Ref & Ref \\
\hline Yes & 1.49 (1.27 to 1.75$)$ & & 1.33 (1.12 to 1.57$)$ & 1.32 (1.10 to 1.57$)$ \\
\hline
\end{tabular}

*Adjusted for sociodemographic characteristics (maternal age, place of residence, education and body mass index).

†Adjusted for obstetric characteristics (antenatal visits, in vitro fertilisation treatment, hypertensive disorder and induction of labour). $\ddagger$ Adjusted for sociodemographic characteristics (age, place of residence, education, body mass index) and obstetric characteristics (antenatal visits, in vitro fertilisation treatment, hypertensive disorder and induction of labour). NA, not applicable due to multicollinearity.

$13.6 \%$ to $31.9 \%$. After adjustment for maternal characteristics, the caesarean section rate still varied from $14.9 \%$ to $32.1 \%$. The main differences in caesarean section rates between trusts appeared with emergency caesarean section, ranging from $10.7 \%$ to $18.9 \%$, compared with elective caesarean section, ranging from $7.8 \%$ to $11.2 \%{ }^{6}$ The authors suggested that the remaining differences in emergency caesarean section across NHS trusts could be due to lack of precise criteria for indications or differences in management practice, which could also be the case in this study.

Another study conducted in Lebanon included 3846 women with singleton, cephalic, viable full-term pregnancy. ${ }^{21}$ The study examined the association between caesarean section and maternal characteristics, pregnancy outcome and characteristics of maternity units. They found large variations between two geographical zones of Lebanon. The authors concluded that the variations between zones were due to variations in patients' access to medical care or variations in clinical practice. ${ }^{21}$ These findings were in line with the findings of this study, but this study focused on the variations between hospitals after adjustment for risk factors.

All hospitals in our study have a neonatal intensive care unit and five of six also have a maternal intensive care unit. Hospital 1 is not a referral hospital and does 
Table 5 OR and 95\% Cls for emergency caesarean section among parous women in the participating hospitals ( $n=24$ 210)

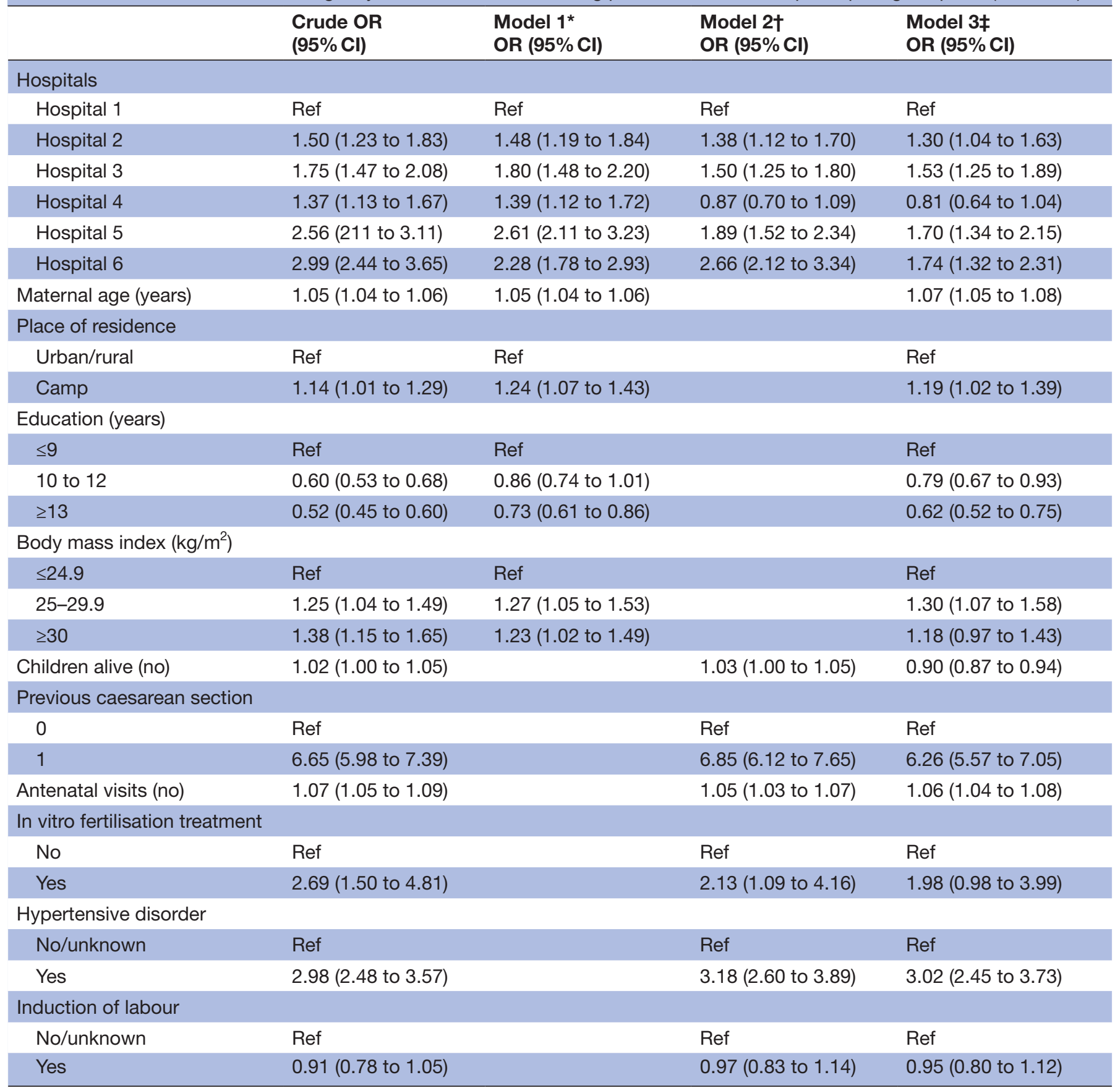

*Adjusted for sociodemographic characteristics (maternal age, place of residence, education and body mass index).

†Adjusted for obstetric characteristics (children alive, previous caesarean section, antenatal visits, in vitro fertilisation treatment, hypertensive disorder and induction of labour).

‡Adjusted for sociodemographic characteristics (age, place of residence, education, body mass index) and obstetric characteristics (children alive, previous caesarean section, antenatal visits, in vitro fertilisation treatment, hypertensive disorder and induction of labour).

not have a maternal intensive care unit for the delivering women. Thus, the lowest emergency caesarean section rate in this hospital may reflect a population with lower risk for complications. All six hospitals were governmental and five of the six were teaching hospitals. The non-teaching one, Hospital 2, had no educational programme for the non-specialist doctors, which may lead to less qualified maternal care. However, this did not increase the odds for emergency caesarean section in this hospital.

A previous study from Norway showed that decision-making for caesarean section delivery by consultants lowered the caesarean section rates. ${ }^{22}$ On call arrangements for each hospital were not explored in this study and the decision-making process for emergency caesarean section may vary across the study hospitals 
due to varying involvement of a specialist in obstetrics during non-office hours. In some of the study hospitals, a specialist in obstetrics is present in the hospital all the time, whereas in others the specialists are available for consultations only and not present in the hospital during the evening and night. Differences in number of consultations, residents and midwives may also affect the decision-making process. However, numbers of consultant, residents and midwives, reported in the previous study in these hospitals, did not have a consistent effect on emergency caesarean section rates. ${ }^{13}$

In Palestine, there are common national guidelines for obstetrics, ${ }^{23}$ but the practical training of midwives and doctors may vary between hospitals. Given the large differences in odds for emergency caesarean section, these guidelines may be applied differently across hospitals due to differences in skills of medical staff causing different use of caesarean section instead of operative vaginal delivery. ${ }^{24}$ A study from the UK, including 216 maternity units, examined the variation in caesarean section rates between delivery units. The study concluded that organisational factors and staffing levels, women's preferences for delivery mode and the clinician's attitudes may explain the variations. ${ }^{25}$ Medical doctors in Palestine are neither insured by their employer nor by the Palestinian Ministry of Health, and may therefore be sued privately if a pregnancy complication occurs. This may also affect their use of emergency caesarean section especially when vaginal births after caesarean section were indicated. It is well known that the decision-makers are affected by their own fear, cultural factors, legal liability and medical evidence. ${ }^{26}$ Also, the fear of perceived risks for complaints and malpractice litigation is associated with requested caesarean section delivery. ${ }^{27}$ In Sweden, caesarean section deliveries for non-medical reasons are $18 \%$ of all caesarean sections, and this rate increased by $80 \%$ from 1990 to $2001 .^{28}$ Physicians' attitudes are known to influence the parents' choice. ${ }^{26}$ However, in governmental Palestinian hospitals, maternal request without medical reason is not a justified indication for caesarean section.

\section{Strengths and limitations of the study \\ Strengths}

The strengths of this study are the population-based approach and the prospective design. The data were collected for research purposes in a prospective manner. All women aiming to give birth vaginally in the six study hospitals were included, reducing the risk for selection bias. A large number of deliveries were included and six different hospitals were compared in Gaza and West Bank for the first time.

\section{Limitations}

The main limitation of this study was the missing data on some deliveries from March 2015 until December 2015. In five of the six study hospitals, less than $4 \%$ of data were missing, but in one hospital, Hospital 6, data were missing for $28 \%$ of its deliveries. ${ }^{13}$ The missing data were expected to be random, not influencing the exposure-outcome associations studied. Data on diabetes before and during pregnancy, known to be associated with increased risk of emergency caesarean section, ${ }^{29}$ were not registered accurately and could therefore not be used for analyses. Moreover, exclusion for multiple pregnancies may have affected the rate of emergency caesarean sections. Since this is a methodological choice, we consider exclusion of multiple pregnancies to be a minor limitation of the study. Additionally, the effect of pre-pregnancy body mass index increased the odds for emergency caesarean section for primiparous women in hospitals except in Hospital 3, which could be due to inaccurate registration of maternal weight. Furthermore, confusion existed among the data collectors in distinguishing rural from urban place of residence. Thus, place of residence was dichotomised as urban/rural versus camp. One further limitation was a lack of data about hospital specification including available resources or staff shift patterns. However, as all study hospitals are governmental hospitals, it is justified to assume that no great differences exist between the hospitals.

\section{CONCLUSION}

Major differences in rates and odds for emergency caesarean section were observed between the six governmental Palestinian hospitals. These could not be explained by differences in the studied sociodemographic or antenatal obstetrical characteristics. These findings may imply that factors related to doctors and their working environments are important in the decision to deliver by emergency caesarean section.

\section{Author affiliations}

${ }^{1}$ Obstetrics Department, Al Shifa Hospital, Gaza, Palestine

${ }^{2}$ Faculty of Medicine, Institute for Clinical Medicine, University of Oslo, Oslo, Norway ${ }^{3}$ Intervention Centre, Oslo University Hospital Rikshospitalet, Oslo, Norway

${ }^{4}$ Department of Obstetrics, Oslo University Hospital, Ullevål, Oslo, Norway

${ }^{5}$ Department of Health Management and Health Economics, Institute of Health and

Society, University of Oslo, Oslo, Norway

${ }^{6}$ Faculty of Pharmacy, Nursing, and Health Professions, Birzeit University, Birzeit, Palestine

${ }^{7}$ Department of Gynecology, Oslo University Hospital, Oslo, Norway

${ }^{8}$ Obstetrics Department, Palestine Medical Complex, West Bank, Palestine

${ }^{9}$ Obstetrics Department, Al Aqsa Hospital, Gaza, Palestine

${ }^{10}$ Biostatistics Department, Oslo School of Management, Oslo, Norway

${ }^{11}$ Faculty of Medicine, Islamic University, Gaza, Palestine

${ }^{12}$ Oslo Centre for Biostatistics and Epidemiology, Research Support Services, Oslo

University Hospital, Oslo, Norway

Acknowledgements The authors would like to extend the best thanks and gratitude to the Norwegian Research Council for funding this study. We do thank the Palestinian Ministry of Health for supporting the study. We also appreciate the effort and time spent by research teams for data collection.

Contributors MZ: in charge of data collection, participated in staff training on data registration and entry, statistical analysis for the data set and drafted the manuscript. KL: study design, protocol and research tool development, participated in staff training on data registration and entry, and drafted the manuscript. SH: study design, collaborated in the preparation of the protocol and research tool development, data collection, participated in staff training on data registration and 
entry, and commented on the manuscript. EF: study design, protocol development and commented on the manuscript. ML: commented on the manuscript. KZ and HA-M: data collection, participated in staff training on data registration and entry, and commented on the manuscript. MA: commented on the manuscript. BB: revised the medical English language and commented on the manuscript. RSF: statistical analysis for the data set and commented on the manuscript. AV: study design, protocol and research tool development, participated in staff training on data registration and entry, and commented on the manuscript. All authors had full access to all the data in the study and can take responsibility for the integrity and accuracy of the data analysis and they had final responsibility for the decision to submit for publication.

Funding This work was supported by the Norwegian Research Council through Oslo University Hospital (234452/14).

Disclaimer The funding source had no role in the study design, data collection, data analysis, data interpretation or writing of the manuscript.

Competing interests None declared.

Patient consent Not required.

Ethics approval This study was approved by the Norwegian Data Inspectorate (17/00082-2/GRA) and the Regional Committee for Medical and Health Research Ethics in South-Eastern Norway and was considered as health quality research (REK 2014/1727). Oslo University Hospital signed an agreement with the Palestinian Ministry of Health, which approved conducting the study within their facilities. The project was done in accordance with common rules for healthcare services in Palestine and Norway regarding confidentiality and privacy.

Provenance and peer review Not commissioned; externally peer reviewed.

Data sharing statement № additional data are available.

Open Access This is an Open Access article distributed in accordance with the Creative Commons Attribution Non Commercial (CC BY-NC 4.0) license, which permits others to distribute, remix, adapt, build upon this work non-commercially, and license their derivative works on different terms, provided the original work is properly cited and the use is non-commercial. See: http://creativecommons.org/ licenses/by-nc/4.0/

(C) Article author(s) (or their employer(s) unless otherwise stated in the text of the article) 2018. All rights reserved. No commercial use is permitted unless otherwise expressly granted.

\section{REFERENCES}

1. Abalos $E$, Addo V, Brocklehurst $P$, et al. Caesarean section surgical techniques: 3 year follow-up of the CORONIS fractional, factorial, unmasked, randomised controlled trial. Lancet 2016;388:62-72.

2. Betrán AP, Ye J, Moller AB, et al. The increasing trend in caesarean section rates: global, regional and national estimates: 1990-2014. PLoS One 2016;11:e0148343.

3. Lumbiganon P, Laopaiboon M, Gülmezoglu AM, et al. Method of delivery and pregnancy outcomes in Asia: the WHO global survey on maternal and perinatal health 2007-08. Lancet 2010;375:490-9.

4. Shorten A. Maternal and neonatal effects of caesarean section. BMJ 2007;335:1003-4.

5. Kwee A, Elferink-Stinkens PM, Reuwer PJ, et al. Trends in obstetric interventions in the Dutch obstetrical care system in the period 1993-2002. Eur J Obstet Gynecol Reprod Biol 2007;132:70-5.

6. Bragg F, Cromwell DA, Edozien LC, et al. Variation in rates of caesarean section among English NHS trusts after accounting for maternal and clinical risk: cross sectional study. BMJ 2010;341:c5065.
7. Librero J, Peiró S, Calderón SM. Inter-hospital variations in caesarean sections. A risk adjusted comparison in the Valencia public hospitals. J Epidemiol Community Health 2000;54:631-6.

8. Habiba M, Kaminski M, Da Frè M, et al. Caesarean section on request: a comparison of obstetricians' attitudes in eight European countries. BJOG 2006;113:647-56.

9. Abdul-Rahim HF, Abu-Rmeileh NM, Wick L. Cesarean section deliveries in the occupied Palestinian territory (oPt): an analysis of the 2006 Palestinian Family Health Survey. Health Policy 2009;93:151-6.

10. Mikki N, Abu-Rmeileh NM, Wick L, et al. Caesarean delivery rates, determinants and indications in Makassed Hospital, Jerusalem 1993 and 2002. East Mediterr Health J 2009;15:868.

11. Ministry of Health. Health Status, Palestine 2015. Ramallah, State of Palestine: Ministry of Health, 2016.

12. van den Berg MM, Madi HH, Khader A, et al. Increasing neonatal mortality among Palestine refugees in the Gaza Strip. PLoS One 2015;10:e0135092.

13. Hassan S, Vikanes A, Laine $\mathrm{K}$, et al. Building a research registry for studying birth complications and outcomes in six Palestinian governmental hospitals. BMC Pregnancy Childbirth 2017;17:1296-6.

14. Jensen DM, Damm P, Sørensen B, et al. Pregnancy outcome and prepregnancy body mass index in 2459 glucose-tolerant Danish women. Am J Obstet Gynecol 2003;189:239-44.

15. Lucas DN, Yentis SM, Kinsella SM, et al. Urgency of caesarean section: a new classification. J R Soc Med 2000;93:346-50.

16. Leone T, Padmadas SS, Matthews Z. Community factors affecting rising caesarean section rates in developing countries: an analysis of six countries. Soc Sci Med 2008;67:1236-46.

17. Pandey S, Shetty A, Hamilton M, et al. Obstetric and perinatal outcomes in singleton pregnancies resulting from IVF/ICSI: a systematic review and meta-analysis. Hum Reprod Update 2012;18:485-503.

18. Deneux-Tharaux C, Carmona E, Bouvier-Colle M-H, et al. Postpartum maternal mortality and cesarean delivery. Obstetrics \& Gynecology 2006;108:541-8.

19. Field A, Haloob R. Complications of caesarean section. The Obstetrician \& Gynaecologist 2016;18:265-72.

20. Lockwood C. Editorial Why the CD rate is on the rise (Part 1). Contemporary Ob Gyn 2004;49:8.

21. Carayol M, Zein A, Ghosn N, et al. Determinants of caesarean section in Lebanon: geographical differences. Paediatr Perinat Epidemiol 2008;22:136-44.

22. Nilsen ST, Bergsjö P, Lökling A, et al. A comparison of cesarean section frequencies in two Norwegian hospitals. Acta Obstet Gynecol Scand 1983;62:555-61.

23. Kurd A, Belbisi A, Jaber S, et al. Palestinian guidelines and protocols for obstetricians, general practitioners and midwives. Preoperative preparation. Ramallah, State of Palestine: Palestinian National Authority, MOH, 2014:191-3.

24. Sachs BP, Kobelin C, Castro MA, et al. The risks of lowering the cesarean-delivery rate. N Engl J Med 1999;340:54-7.

25. Paranjothy S, Frost $C$, Thomas J. How much variation in CS rates can be explained by case mix differences? BJOG: An International Journal of Obstetrics \& Gynaecology 2005;112:658-66.

26. Belizan JM, Althabe F, Barros FC, et al. Rates and implications of caesarean sections in Latin America: ecological study. BMJ 1999;319:1397-402.

27. Fuglenes D, Oian P, Kristiansen IS. Obstetricians' choice of cesarean delivery in ambiguous cases: is it influenced by risk attitude or fear of complaints and litigation? Am J Obstet Gynecol 2009;200:48.e1-48. e8.

28. Elvander C, Dahlberg J, Andersson G, et al. Mode of delivery and the probability of subsequent childbearing: a population-based register study. BJOG 2015;122:1593-600.

29. Ehrenberg HM, Mercer BM, Catalano PM. The influence of obesity and diabetes on the prevalence of macrosomia. Am J Obstet Gynecol 2004;191:964-8. 